\title{
EFFECT OF ADDING EQUEX STM PASTE AND BHT TO DIFFERENT EXTENDERS ON VIABILITY, PLASMA MEMBRANE AND DNA INTEGRITY OF GERMAN SHEPHERD DOG SPERMATOZOA DURING FREEZING AND THAWING METHODS IN EGYPT
}

\author{
MARY G. ABDEL- MALAK ${ }^{*}$; MAHMOUD, A.S. ${ }^{* *}$; HUSSEIN, F.M. ${ }^{* * *}$ and METWALLY, K.K. ${ }^{* * *}$ \\ *Artificial Insemination and Embryo Transfer Dep., Animal Reproduction Research Institute, Al Haram, Giza, Egypt. \\ *** Veterinarian in Egyptian Armed Force. \\ *** Theriogenology Dep. Faculty of Veterinary Medicine, Alexandria University, Egypt.
}

\section{ABSTRACT}

Received at: 30/3/2015

Many factors influencing semen quality through all steps of cryopreservation such as semen processing, the combination of extender, cooling rate during the freezing

Accepted: 30/4/2015 procedure and the thawing technique. The present study was conducted to evaluate the potential cryoprotective effect of Equex STM paste and / or butylated hydroxytoluene (BHT) occlusion in Tris and milk based extenders, freezing according to 2 methods, thawing at 2 regimes, and the interactions between these treatments, on the post-thaw semen quality. A total number of 80 ejaculates was collected from 15 German shepherd stud dogs. Semen samples was frozen in two different extenders, Tris (TCF) and milk (SMG) based extenders, with or without $0.5 \%$ Equex STM paste and / or $1 \mathrm{mM}$ BHT compared with commercial bovine tris based semen extender (BULLXcell ${ }^{\mathrm{TM}}$, IMV technologies, Franc) using 2 freezing protocols $(4$ and $6 \mathrm{~cm})$ above $\mathrm{LN}_{2}$ and 2 thawing rates $\left(45^{\circ} \mathrm{C}\right.$ for $30 \mathrm{sec}$. and $55^{\circ} \mathrm{C}$ for $5 \mathrm{sec}$.). Post-thaw motility, viability, plasma membrane integrity in terms of post-thaw live spermatozoa, acrosomal defects, acrosome reaction and rate of increase in activity of extracellular AST, ALT and ALP enzymes as well as assessment of sperm DNA integrity using comet assay were recorded. The results clarified that addition of Equex STM paste and / or BHT to the TCF and SMG extenders significantly $(\mathrm{P}<0.01)$ improved post- thaw motility, viability, plasma membrane integrity and reduced acrosomal defects, acrosomal reaction, release of extracellular AST, ALT and ALP enzymes and DNA damage. The best post-thaw semen qualities was observed when freezing in a Styrofoam box above $6 \mathrm{~cm}$ highest above $\mathrm{LN}_{2}$ and thawing at $55{ }^{\circ} \mathrm{C}$ for $5 \mathrm{sec}$. the superior results were obtained with Tris based extenders than milk based extenders. It is concluded that fortification of $0.5 \%$ Equex STM paste and / or $1 \mathrm{mM}$ BHT in Tris based extenders freezing above 6 $\mathrm{cm}$ highest above $\mathrm{LN}_{2}$ and thawing at $55{ }^{\circ} \mathrm{C}$ for $5 \mathrm{sec}$. can improve the post-thaw dog semen motility, viability and plasma membrane integrity. Also, the commercial bovine extender BULLXcell ${ }^{\mathrm{TM}}$ is useful for cryopreservation of dog semen.

Keywords: Equex STM paste, BHT, BULLXcell ${ }^{\mathrm{TM}}$, canine, DNA, acrosome.

\section{INTRODUCTION}

Cryopresrvation of semen has become a valuable tool for the preservation of genetic reserves of endangered species or sires of superior breeding value. This technique is routinely used for bovine, ovine and equine sperm and interest is increasing for canine species. Many dog breeders wish to improve their breeding results with foreign blood lines, but while it is getting easier to ship cryopreserved semen, fertilizing results after insemination with frozenthawed semen are still variable (Linde-Forsberg et al., 1999). The exchange of canine frozen semen among many countries is widely applied; therefore an increase in the number of artificial insemination banks has been noticed. Rowson (1954) was the first researcher to freeze canine semen, and then many studies were developed to find a good extender and cryoprotectant to preserve canine semen.

German shepherd dog is known for termagancy, well trained and natural police dogs. China has set up the breeding system of German shepherd dog which completely adapted to need of the public security, troops and the national defense on the preservation, breeding and utilization (Wang et al., 2008). Pan 
et al. (1986) firstly researched the semen freezing and AI of German shepherd dogs and succeeded. Also, Wang et al. (1991) succeeded with semen freezing of German shepherd dog too and obtained higher pregnancy rate and litter size.

Many factors influencing semen quality through all steps of cryopreservation such as technique of semen collection, the extender and the final concentration of spermatozoa (Okano et al., 2004) semen processing, the combination of extender and cooling rate during the freezing procedure (Rota et al., 1998) and the thawing technique (Peña and Linde-Forsberg 2000a).

The cryopreservation process is accompanied by excessive generation of reactive oxygen species (ROS) which may induce changes in the structure and functions of the sperm plasma membrane (Wang et al., 1997). To overcome the deleterious effects of ROS is the addition of various antioxidants to the freezing extenders, which improves the semen quality after thawing. Butylated hydroxytoluene (BHT) is one such example being tested for its cryoprotective potentiality. BHT has been used successfully for minimizing cryoinjury in ram (Watson and Anderson, 1983), boar (Roca et al., 2004), cattle bull (Shoae and Zamiri, 2008), and goat spermatozoa (Khalifa et al., 2008). However, there are scanty reports (Neagu et al., 2010; Sahashi et al., 2011 and Ziaullah et al., 2012) regarding its cryoprotective potentiality and optimal inclusion level in canine semen.

Also, from the best membrane stabilizing substance is Equex STM paste when adding to semen extenders in $0.5 \%$ just before freezing (Schäfer-Somi et al., 2006) has shown beneficial effects on frozen-thawed dog semen with regard to post-thaw motility, thermoresistance, increased longevity and plasma membrane integrity (Rota et al., 1997; Peña and Linde-Forsberg 2000b).

In Egypt, there is no literature concerning the role of Equex STM paste and BHT, different freezing protocols as well as different thawing regimes in preservation of German shepherd dog semen. So, The objectives of the present study were to evaluate the effect of Equex STM paste and / or BHT occlusion in different extenders, freezing according to 2 methods, thawing at 2 regimes, and the interactions between these treatments, on the post-thaw motility, viability, plasma membrane as well as DNA integrity to improve breeding efficiency of male dogs by prolonging semen gametes preservation.

\section{MATERIALS and METHODS}

This work was resulted from the cooperation between Animal Reproduction Research Institute (A.R.R.I) Al-Haram, Giza, Faculty of Veterinary Medicine,
Alexandria University and Egyptian Armed Force kennel. This study started at November 2012.

\section{Animals}

Fifteen healthy, proven fertility after natural mating stud dogs German shepherd with an average of 1.5-3 years of age and average body weight $34.94 \mathrm{Kg}$ $(27 \sim 42.5 \mathrm{~kg})$ were used.

\section{Reagents}

All chemicals were from Sigma Aldrich (USA) except tris, glucose, fructose and citric acid monohydrate, which from Oxford laboratory reagent (Mumbai, India) and Glycerol from (Fisher bioreagents).

\section{Semen Extenders}

Two chemically defined extenders, Tris- citric acid fructose (TCF) according to Alamo et al. (2005) and skim milk-glucose (SMG) according to Baran et al. (2000) were used in this study comparing with commercial bovine semen extender BULLXcell ${ }^{\mathrm{TM}}$ (IMV technologies, France). Tris based extenders, and milk based extenders were prepared as declared in Table (1\&2), while BULLXcell ${ }^{\mathrm{TM}}$ extender was commercial bovine semen extender (IMV technologies, France), which consists of tris, citric acid, sugars, glycerol, ultra-pure water, antibiotics (Gentamycin, Tylosin, lincomycin and Spectinomycin). BULLXcell ${ }^{\mathrm{TM}}$ extender was prepared with 1 part of BULLXcell ${ }^{\mathrm{TM}}$ liquid, 1 part of egg yolk and 3 parts pure distilled water as described in the instruction sheet of IMV technologies company, France. The final $\mathrm{pH}$ and osmolarity after preparation were 6.8 and $1.141 \quad \mathrm{osmol} / \mathrm{kg}$ respectively.

$1 \mathrm{mM}$ BHT dissolved in $1 \mathrm{ml}$ DMSO (Neagu et al., 2010) and Equex STM paste $(0.5 \%)$ according to Farstad (2012) were added as additives to (TCF) and (SMG) extenders as shown in Table (1\&2). Finally, 9 extenders in this study were used. $\mathrm{pH}$ was adjusted at 6.7 in all extenders except BULLXcell ${ }^{\mathrm{TM}}$ using $\mathrm{pH}$ meter (Adwa, Romania, Europe). Osmolarity were measured using cryoscopic osmometer (OSMOMAT 030, Gonotec Company).

\section{Semen collection and processing}

Semen was collected by digital manipulation (Kutzler, 2005) in the presence of estrus bitch with a $72 \mathrm{hr}$ interval between the collections using rubber silicon cone (Linde Forsberg, 2005). Only the $2^{\text {nd }}$ sperm-rich fraction $(0.5-3 \mathrm{ml})$ of the ejaculate was used. A total number of 80 ejaculates was used for this study. Immediately after collection, the semen samples were evaluated for volume, motility, sperm concentration and total sperm abnormalities. Only semen samples of at least $75 \%$ initial motility, 400 $\mathrm{x} 10^{6}$ sperm cell $/ \mathrm{ml}$ and total abnormal sperm $\leq 20 \%$ were regarded suitable for freezing. 
The semen samples were pooled in order to have sufficient semen to replicate and eliminate the individual effect (Michael et al., 2007) and divided into 3 aliquots. Each aliquot diluted $1: 1(\mathrm{v} / \mathrm{v})$ at room temperature $\left(25^{\circ} \mathrm{C}\right)$ to reach $200 \times 10^{6}$ sperm cell/ ml with TCF, SMG and BULLXcell ${ }^{\mathrm{TM}}$. The diluted samples were transferred to the laboratory throughout one hour by animal semen dry shipper box (Neopor box) to reach gradually $13^{\circ} \mathrm{C}\left(0.2^{\circ} \mathrm{C} / 1 \mathrm{~min}\right)$ during transportation, then cooled to $5^{\circ} \mathrm{C}$ over a period of 1hour and equilibrated for $1 \mathrm{hr}$. The semen samples were then loaded in $0.25 \mathrm{ml}$ PVC ministraws (IMV, L, Aigle, France) and arranged horizontally on freezing racks at $5^{\circ} \mathrm{C}$ before suspended in liquid nitrogen $\left(\mathrm{LN}_{2}\right)$ vapor inside a closed Styrofoam box $\left(54 \times 35 \times 18 \mathrm{~cm}\right.$, containing 10 liters $\left.\mathrm{LN}_{2}\right)$ for $15 \mathrm{~min}$ at two freezing rates $(4$ and $6 \mathrm{~cm}$ ) above the surface of $\mathrm{LN}_{2}$ and plunged directly in it. After one week of storage, the straws were thawed by removing 2 straws from $\mathrm{LN}_{2}$ and dropping them in water bath at two thawing regimes $\left(45^{\circ} \mathrm{C}\right.$ for $30 \mathrm{sec}$. and $55^{\circ} \mathrm{C}$ for 5 sec.). The straws were wiped dry after thawing and deplugged by cutting off with scissors, then transferred into prewarmed, clean, narrow glass $2 \mathrm{ml}$ test tubes and incubated in an incubator adjusted at 37 ${ }^{\circ} \mathrm{C}$ for 3 hours. The frozen-thawed semen parameters were evaluated (Exp.1).

While, in Exp.2, the collected semen samples were divided into 9 aliquots and each aliquot diluted $1: 1(\mathrm{v} / \mathrm{v})$ at room temperature $\left(25{ }^{\circ} \mathrm{C}\right)$ to reach 200 $\mathrm{x} 10^{6}$ sperm cell/ $\mathrm{ml}$ with 8 chemically defined extenders previously mentioned in Tables $1 \& 2$ before further extended with, an equal volume of extenders (2, 4, 6 and 8), 1\% Equex STM containing extender to reach final concentration $0.5 \%$ Equex STM just before freezing (two step dilution) while the $9^{\text {th }}$ aliquot was diluted in BULLXcell ${ }^{\mathrm{TM}}$. The diluted samples were transferred to the lab., cooled, equilibrated and freezing at $6 \mathrm{~cm}$ above the surface of $\mathrm{LN}_{2}$ and plunged directly in it as mentioned previously in Exp (1). After one week of storage, the straws were thawed in water bath at $55^{\circ} \mathrm{C}$ for $5 \mathrm{sec}$. The frozen-thawed semen parameters were evaluated.

Table 1: Tris based extenders

\begin{tabular}{ccccc}
\hline Ingredients & $\begin{array}{c}\text { Ext. 1 } \\
\text { (TCF) } \\
\text { Control }\end{array}$ & $\begin{array}{c}\text { Ext. 2 } \\
\text { (TCF- Eq) }\end{array}$ & $\begin{array}{c}\text { Ext. 3 } \\
\text { (TCF-BHT) }\end{array}$ & $\begin{array}{c}\text { Ext. 4 } \\
\text { (TCF-Eq-BHT) }\end{array}$ \\
\hline $\begin{array}{c}\text { Tris (g) } \\
\text { (Hydroxy methyl aminomethane) }\end{array}$ & 3.025 & 3.025 & 3.025 & 3.025 \\
\hline Citric acid monohydrate (g) & 1.7 & 1.7 & 1.7 & 1.7 \\
\hline Fructose (g) & 1.25 & 1.25 & 1.25 & 1.25 \\
\hline Streptomycin (g) & 0.10 & 0.10 & 0.10 & 0.10 \\
\hline BenzyLpenicillin (U/mL) & 100.000 & 100.000 & 100.000 & 100.000 \\
\hline Ultrapure water (ml) & To & To & To & $100 \mathrm{ml}$ \\
\hline Egg yolk (ml) & $100 \mathrm{ml}$ & $100 \mathrm{ml}$ & $100 \mathrm{ml}$ & 20 \\
\hline Glycerol (ml) & 20 & 20 & 20 & 5 \\
\hline Equex paste (ml) & 5 & 5 & 5 & 1.00 \\
\hline BHT (mM) & 0 & 1.00 & 0 & 6.76 \\
\hline PH & 0 & 0 & 6.76 & 1.546 \\
\hline Osmolarity (osmol/kg) & 1.750 & 1.534 & 1.543 & \\
\hline
\end{tabular}

Table 2: Milk based extenders

\begin{tabular}{ccccc}
\hline Ingredients & $\begin{array}{c}\text { Ext. 5 } \\
\text { (SMG) } \\
\text { Control }\end{array}$ & $\begin{array}{c}\text { Ext. 6 } \\
\text { (SMG - Eq) }\end{array}$ & $\begin{array}{c}\text { Ext. 7 } \\
\text { (SMG -BHT) }\end{array}$ & $\begin{array}{c}\text { Ext. 8 } \\
\text { (SMG -Eq-BHT) }\end{array}$ \\
\hline Glucose (g) & 1.00 & 1.00 & 1.00 & 1.00 \\
\hline Streptomycin (g/mL) & 0.10 & 0.10 & 0.10 & 0.10 \\
\hline BenzyL penicillin (U/mL) & 100.000 & 100.000 & 100.000 & 100.000 \\
\hline Skim-Milk to final volume & To & To & To & 100 ml \\
\hline Egg yolk (ml) & $100 \mathrm{ml}$ & $100 \mathrm{ml}$ & $100 \mathrm{ml}$ & 20 \\
\hline Glycerol (ml) & 20 & 20 & 20 & 5 \\
\hline Equex paste (ml) & 5 & 5 & 5 & 1.00 \\
\hline BHT (mM) & 0 & 1.00 & 0 & 6.76 \\
\hline PH & 0 & 0 & 1.00 & 1.277 \\
\hline Osmolarity $(\mathbf{o s m o l} / \mathbf{k g})$ & 6.76 & 6.76 & 6.76 & 1.280 \\
\hline
\end{tabular}


Examination of frozen-thawed semen

Sperm motility (\%) was subjectively assessed immediately after dilution, before freezing and after thawing by using a phase-contrast microscope (40x) equipped with a thermal stage at $37^{\circ} \mathrm{C}$. Also, sperm motility was reassessed after 1,2 and 3 hours of thawing and post-thaw viability index was calculated according to Milovanov (1962). The post-thaw recovery rate $(\%)$ was calculated to the following formula:

Sperm motility percentage immediately after thawing

Sperm motility percentage immediately after dilution

The rate of reduction in post-thaw sperm motility (\%) was calculated to the following formula:

Percent motile sperm at $0 \mathrm{hr}-$ Percent motile sperm at $3 \mathrm{hr}$

Percent motile sperm at $0 \mathrm{hr}$

$-\mathrm{X} 100$

Sperm plasma membrane integrity was assessed immediately after thawing by using:

1-Eosin stain exclusion assay (Vazquez et al., 1997) for assessment of post-thaw live spermatozoa (\%) and acrosomal defects $(\%)$.

2-Acrosome reaction (\%) using silver nitrate stain according to El-Amrawi and Nemetalla (1991).

3-leakage of intracellular enzymes (Aspartate aminotransferase (AST/GOT), Alanine aminotransferase (ALT/GPT) and alkaline phosphates (ALP) Enzymes into the extracellular medium:-

During freeze- thaw processing of all split semen samples, $0.5 \mathrm{ml}$ of diluted semen was taken immediately before freezing and immediately after thawing. The diluted and thawed semen were then centrifuged at $700 \mathrm{xg}$ for $15 \mathrm{~min}$. at $30^{\circ} \mathrm{C}$ for separation of seminal plasma. The collected seminal plasma was stored at $-20^{\circ} \mathrm{C}$ until determination of AST, ALT and ALP enzymes. These enzymes were assessed spectrophotometrically using Modular analytics P800 analyzer using commercial kits from cobas ${ }^{\circledR}$ (Roche, Hitachi, GmbH, Germany).

Determinations of AST and ALT were performed by Colorimetric method according to Reitman and Frankel (1957); and Young (1990). While, ALP were performed by Colorimetric method of Belfield and Goldberg (1971). The results were expressed in terms of units per liter (U/L). The rate of increase in postthaw activity of extracellular of each enzyme (\%) was calculated according to the following formula:

Post-thaw enzyme activity - pre-freeze enzyme activity X 100

Pre-freeze enzyme activity
4- Assessment of sperm DNA integrity using Single cell gel electrophoresis (SCGE) comet assay:

The alkaline comet assay for DNA damage in spermatozoa was carried out according to Hughes et al. (1997) with some modifications DNA damage was detected in semen samples by centrifugation of diluted semen sample at $3000 \mathrm{rpm} / 10 \mathrm{~min}$. and resuspended in the sperm culture medium (S-TALP buffer) and stored at $-20{ }^{\circ} \mathrm{C}$ until analyzed. The DNA integrity and the incidence of DNA strand breaks or fragmentation was detected using alkaline comet assay according to (Singh et al., 1988). The sperm comets were visually scored according to Collines et al. (1995).

\section{Statistical analysis:}

It can be done by using a computerized Statistical analysis system (Costat 1986).

\section{RESULTS}

Exp.1: Effect of freezing rates as well as thawing regimes on motility and viability of frozen- thawed dog spermatozoa:

Regarding to the thawing regime at $45^{\circ} \mathrm{C}$ for $30 \mathrm{sec}$, Table (3) recorded the overall means percentages of sperm motility after thawing $(53.50 \pm 2.02,49.25$ \pm 4.47 and $35.00 \pm 1.49 \%$ ) in BULLXcell, TCF and SMG extenders respectively. The overall mean values of post-thaw viability indices in 3 previous extenders were 90.25 $\pm 7.36,73.13 \pm 5.27$ and $28.75 \pm 1.85$ respectively. The maximum significant $(\mathrm{P}<0.01)$ values of viability indices were recorded with BULLXcell followed by TCF then SMG extenders. The overall means of calculated post-thaw recovery rate in different three above extenders were $65.75 \pm$ $2.54,61.03 \pm 2.33$ and $45.79 \pm 2.06 \%$ respectively (Table 5). Regardless the effect of semen extenders in Tables (3 and 5), the mean values of post-thaw sperm motility, viability index and recovery rate were evidently improved after freezing of dog semen above liquid nitrogen surface by $6.00 \mathrm{~cm}(48.66 \pm 2.22 \%$, $70.83 \pm 7.31$ and $61.12 \pm 2.75 \%$, respectively) than those above liquid nitrogen surface by $4.00 \mathrm{~cm}$ $(43.16 \pm 1.68 \%, 57.25 \pm 5.19$ and $53.93 \pm 1.90 \%$, respectively). Analysis of variance revealed highly significant effect for both semen extenders and heights of dog semen above liquid nitrogen surface on post-thaw sperm motility $(\mathrm{P}<0.01)$, viability index $(\mathrm{P}<0.05)$ as well as post-thaw recovery rate $(\mathrm{P}<$ 0.01). No significant interaction between semen extenders and liquid nitrogen heights was observed. Regarding to second thawing regime at $55^{\circ} \mathrm{C}$ for $5 \mathrm{sec}$ using BULLXcell, (TCF) and (SMG) extenders, the overall means percentages of sperm motility were $(57.25 \pm 1.22 \& 52.00 \pm 0.76$ and $29.00 \pm 2.50 \%)$ in 3 above extenders respectively (Table 4). The maximum values of viability indices were recorded with BULLXcell $(117.37 \pm 6.67)$ and TCF (119.75 
$\pm 4.65)$ followed by SMG $(38.25 \pm 6.33)$ extenders. While, the maximum values of calculated post-thaw recovery rate were recorded with BULLXcell (70.69 \pm $1.84 \%)$ followed by TCF $(64.03 \pm 0.91 \%)$ then SMG $(37.09 \pm 3.10 \%)$ extenders respectively (Table 5). Regarding to the effect of heights of dog semen above liquid nitrogen surface the results obtained were parallel with the results of the first thawing regime in Tables 4 and 5, the mean values of post-thaw sperm motility, viability index and recovery rate were also evidently improved after freezing of dog semen above liquid nitrogen surface by $6.00 \mathrm{~cm}(49.00 \pm 2.14 \%$ and $106.50 \pm 7.57$, Table 4$)$ and $(61.17 \pm 2.61 \%$, Table 5) respectively, than those above liquid nitrogen surface by $4.00 \mathrm{~cm}(43.16 \pm 2.96 \%, 77.08 \pm 8.54$, Table 4) and (53.36 $\pm 3.56 \%$, Table 5) respectively. Analysis of variance revealed a highly significant $(\mathrm{P}<$ 0.01 ) effect for both semen extenders and heights of dog semen above liquid nitrogen surface on post-thaw sperm motility, viability index as well as post-thaw recovery rate. Also, there was a highly significant $(\mathrm{P}<$ 0.01 ) interaction between semen extenders and liquid nitrogen heights in post-thaw motility and recovery rate parameters. While, no significant interaction in viability index was observed.

Exp.2: Effect of Equex STM paste and BHT on viability and membrane integrity of dog sperm.

This experiment was conducted for declaring the influence of 2 chemically defined extenders (TCF and SMG) with or without 3 additives (Equex STM paste and/ or BHT) comparing with BULLXcell (commercial bovine extender) on the motility, viability and plasma membrane integrity throughout the different stages of freeze/ thaw processing of dog semen.

Sperm motility after thawing at $55^{\circ} \mathrm{C}$ for $5 \mathrm{sec}$, viability index and post-thaw recovery rate (Table 6) were significantly $(\mathrm{P}<0.01)$ improved by supplementation of tris based extender with $0.5 \%$ Equex STM paste (TCF- Eq) and/or 1mM BHT (TCF- BHT) and TCF-Eq+BHT (61.50 $\pm 1.29,59.00$ \pm 1.63 and $61.00 \pm 1.24 \%$ respectively) and (175.25 $\pm 8.31, \quad 158.00 \pm 3.45$ and $175.00 \pm 7.53)$ respectively. Regarding to post-thaw recovery rate, the maximum percentage was recorded with TCFEq+BHT $(77.82 \pm 3.77 \%)$ than other treated and control samples. In the same line, the bovine commercial extender (BULLXcell) significantly improved post-thaw motility (56.00 $\pm 2.08 \%)$, viability index $(134.00 \pm 6.68)$ and recovery rate $(68.28 \pm 2.32 \%)$. With respect to the fortification of milk based extender with the same previous additives, the only significant $(\mathrm{P}<0.01)$ improvement of postthaw motility $(42.00 \pm 2.70 \%)$, viability index $(84.00 \pm$ $9.78)$ and post-thaw recovery rate $(53.62 \pm 2.93 \%)$ was observed in milk based extender supplemented with $0.5 \%$ Equex STM paste (SMG- Eq).
Table 7 highlights the influence of different extenders and additives on the capability of frozen- thawed sperm to exclude the eosin stain as a reliable criterion for sperm plasma membrane integrity. It was evident that fortification of tris based extender with $0.5 \%$ Equex STM paste (TCF- Eq) significantly $(\mathrm{P}<0.01)$ increased post-thaw percentage of unstained spermatozoa $(67.90 \pm 0.68 \%)$ followed by BULLXcell extender $(60.80 \pm 1.25 \%)$ then TCF-Eq+ BHT $(56.90 \pm 1.53 \%)$ than the other 6 extenders. Also, supplementation of tris based extender with Equex STM paste alone or with BHT as well as commercial bovine extender (BULLXcell) resulted in a highly significantly $(\mathrm{P}<0.01)$ reduction in the incidence of post-thaw acrosomal defects and acrosome reaction (complete and incomplete). The minimum percentages of acrosoml defects in the three above mentioned extenders were $(36.30 \pm 0.39,39.30 \pm 1.71$ and $36.80 \pm 1.87 \%$, respectively). Also, the minimum percentages in complete and incomplete acrosome reaction were $(8.80 \pm 0.32 \& 11.80 \pm 0.66 \%),(11.00 \pm$ $0.25 \% \& 17.50 \pm 0.16 \%)$ and $(9.40 \pm 0.30 \& 17.50 \pm$ $0.16 \%$ ) respectively than other 6 extenders.

Table (8) reveals the influence of extenders and additives on the stability of sperm plasma membrane immediately after thawing of cryopreserved dog semen. It was clear that the commercial bovine extender (BULLXcell) and fortification of tris and milk based extender with either Equex STM paste alone or with BHT significantly $(\mathrm{P}<0.01)$ minimized the rate of increase in post-thaw activity of extracellular AST, ALT and ALP enzymes. The minimum rate of increase in post-thaw activity of extracellular AST, ALT and ALP enzymes were recorded in TCF- $\mathrm{Eq}+$ BHT $(29.60 \pm 1.79$, $38.50 \pm 4.53$ and $23.00 \pm 1.54 \%)$, TCF- Eq (38.10 \pm $2.43,41.70 \pm 1.07$ and $32.20 \pm 2.44 \%$ ), BULLXcell $(30.20 \pm 0.90,48.65 \pm 2.17$ and $55.60 \pm 1.80 \%)$, SMG- Eq $(42.30 \pm 2.58,46.00 \pm 1.63$ and $49.50 \pm$ $1.38 \%)$ and SMG - Eq+ BHT (43.60 \pm 1.42 , $49.60 \pm 3.03$ and $46.50 \pm 1.38 \%$ ) respectively.

\section{Assessment of sperm DNA integrity using comet assay.}

Table (9) reported the influence of extenders and additives on the DNA integrity immediately after thawing of cryopreserved dog semen. Concerning the percentages of DNA damage, fortification of tris based extender with Equex STM paste and BHT significantly $(\mathrm{P}<0.01)$ minimized the post-thaw percentages of DNA damage (11.95 $\pm 0.02 \%)$, while, the maximum value was recorded with milk based extender $(29.00 \pm 1.73 \%)$. No significant difference between the other seven extenders was observed.

Regarding to the tail length and tail moment, commercial bovine extender (BULLXcell) and fortification of tris based extender with Equex STM 
paste plus BHT significantly $(\mathrm{P}<0.01)$ improved membrane integrity of post-thaw dog sperm in terms of tail length $(8.10 \pm 0.46 \& 9.20 \pm 0.28 \mu \mathrm{m}$, respectively) and tail moment (1.60 $\pm 0.23 \& 2.05$ \pm 0.14 Unit, respectively) followed by tris based extenders fortified with Equex STM paste (12.60 $\pm 2.13 \mu \mathrm{m} \& 4.75 \pm 0.02$ Unit, respectively) or with BHT $(12.65 \pm 0.31 \mu \mathrm{m} \quad \& \quad 4.85 \pm 0.14 \quad$ Unit, respectively) as well as milk based extender supplemented with Equex STM paste $(14.00 \pm 1.38$ $\mu \mathrm{m} \& 4.90 \pm 1.50$ Unit, respectively). While, the significant maximum value of tail length and tail moment was recorded with milk based extender (18.35 $\pm 0.25 \mu \mathrm{m} \& 7.40 \pm 0.34$ Unit) respectively. Analysis of variance reveled a highly significantly $(\mathrm{P}<0.01)$ effects of semen treatments on all measurements of DNA integrity.

Table 3: Effect of heights of dog semen above $\mathrm{LN}_{2}$ surface on post-thaw sperm motility (\%) and viability index at thawing regime $45{ }^{\circ} \mathrm{C}$ for $30 \mathrm{sec}$ in different extenders (Mean $\pm \mathrm{SE}$ ).

\begin{tabular}{|c|c|c|c|c|c|c|}
\hline \multirow[b]{2}{*}{ tet } & \multicolumn{2}{|c|}{ Post-thaw motility } & \multirow{2}{*}{$\begin{array}{c}\text { Overall } \\
\text { Means }\end{array}$} & \multicolumn{2}{|c|}{ Viability index } & \multirow{2}{*}{$\begin{array}{c}\text { Overall } \\
\text { Means }\end{array}$} \\
\hline & $4 \mathrm{~cm}$ & $6 \mathrm{~cm}$ & & $4 \mathrm{~cm}$ & $6 \mathrm{~cm}$ & \\
\hline \multirow{2}{*}{ BULLXcell } & 50.00 & 57.00 & $53.50^{\mathrm{A}}$ & 76.50 & 104.00 & $90.25^{A}$ \\
\hline & $1.25 \pm$ & $\pm \mathbf{3 . 0 8}$ & \pm 2.02 & $7.02 \pm$ & \pm 11.73 & \pm 7.36 \\
\hline \multirow{2}{*}{ TCF } & 45.50 & 53.00 & $49.25^{A}$ & 65.25 & 81.00 & $73.13^{B}$ \\
\hline & \pm 1.73 & \pm 2.37 & \pm 4.47 & \pm 8.60 & \pm 5.43 & \pm 5.27 \\
\hline \multirow{2}{*}{ SMG } & 34.00 & 36.00 & $35.00^{B}$ & 30.00 & 27.50 & $28.75^{C}$ \\
\hline & \pm 2.08 & \pm 0.22 & \pm 1.49 & \pm 2.60 & \pm 2.71 & \pm 1.85 \\
\hline Overall means & $\begin{array}{c}4^{43.16}{ }^{b} \\
\pm 1.68\end{array}$ & $\begin{array}{c}48.66^{\mathrm{a}} \\
\pm 2.22\end{array}$ & & $\begin{array}{c}\mathbf{5 7 . 2 5}^{\mathbf{b}} \\
\pm \mathbf{5 . 1 9}\end{array}$ & $\begin{array}{c}\mathbf{7 0 . 8 3}^{\mathrm{a}} \\
\pm 7.31\end{array}$ & \\
\hline
\end{tabular}

Means with different superscripts $\mathrm{A}, \mathrm{B}, \mathrm{C}$ between rows and $\mathrm{a}, \mathrm{b}$ between columns are significantly different at least $(\mathrm{P}<0.01)$.

Table 4: Effect of heights of dog semen above $\mathrm{LN}_{2}$ surface on post-thaw sperm motility (\%) and viability index at thawing regime $55{ }^{\circ} \mathrm{C}$ for $5 \mathrm{sec}$ in different extenders (Mean $\pm \mathrm{SE}$ ).

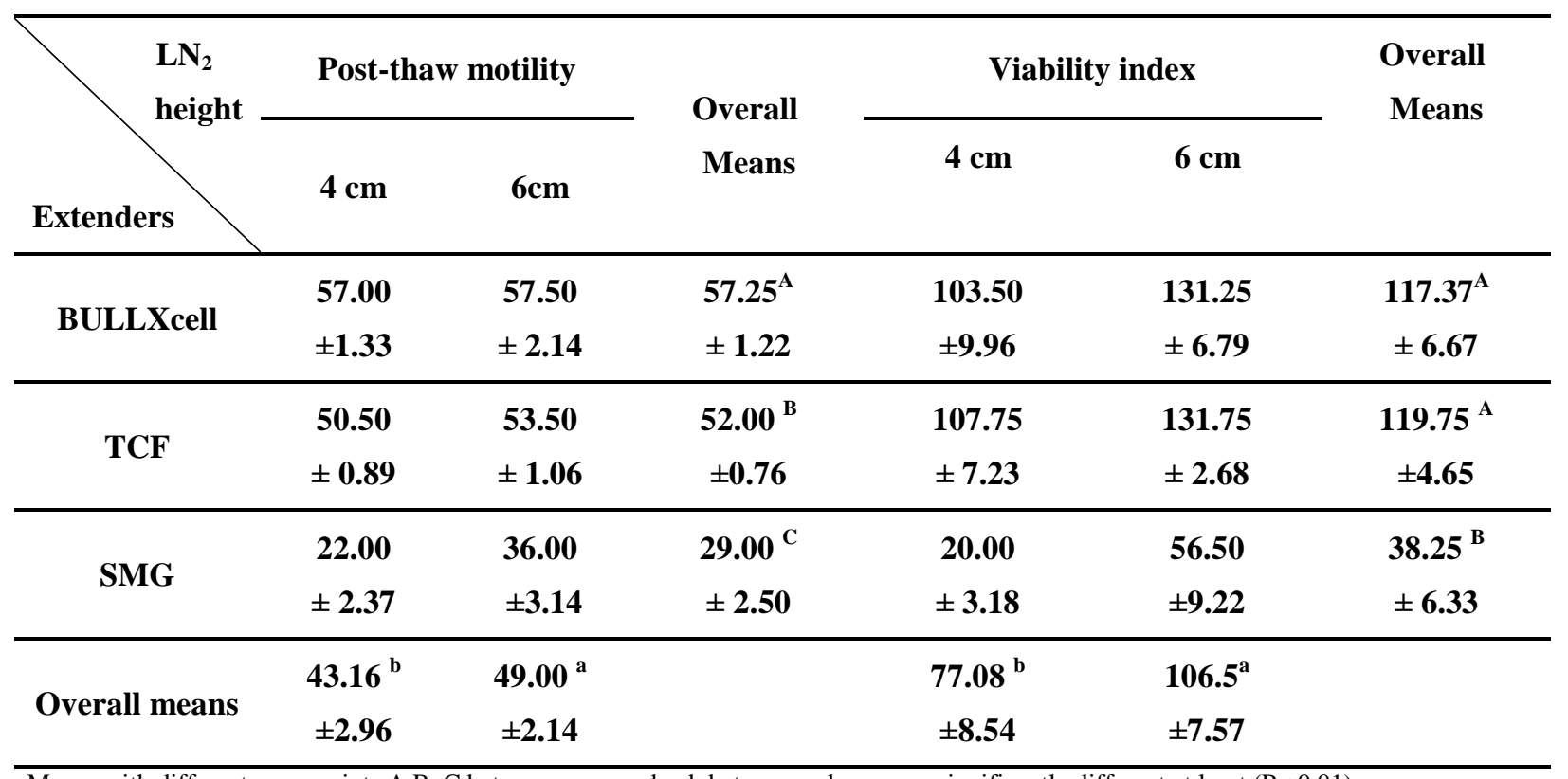

Means with different superscripts $\mathrm{A}, \mathrm{B}, \mathrm{C}$ between rows and $\mathrm{a}, \mathrm{b}$ between columns are significantly different at least $(\mathrm{P}<0.01)$. 
Table 5: Effect of heights of dog semen above $\mathrm{LN}_{2}$ surface on post-thaw recovery rate $(\%)$ at thawing regime $45^{\circ} \mathrm{C}$ for $30 \mathrm{sec}$ and $55^{\circ} \mathrm{C}$ for $5 \mathrm{sec}$ in different extenders (Mean $\pm \mathrm{SE}$ ).

\begin{tabular}{|c|c|c|c|c|c|c|}
\hline \multirow[b]{2}{*}{ Extenders } & \multicolumn{2}{|c|}{$\begin{array}{l}\text { Post-thaw } \\
\text { recovery rate }(\%) \\
\text { at } 45^{\circ} \mathrm{C} \text { for } 30 \mathrm{sec}\end{array}$} & \multirow{2}{*}{$\begin{array}{c}\text { Overall } \\
\text { Means }\end{array}$} & \multicolumn{2}{|c|}{$\begin{array}{c}\text { Post-thaw } \\
\text { recovery rate }(\%) \\
\text { at } 55^{\circ} \mathrm{C} \text { for } 5 \mathrm{sec}\end{array}$} & \multirow[t]{2}{*}{$\begin{array}{c}\text { Overall } \\
\text { Means }\end{array}$} \\
\hline & $4 \mathrm{~cm}$ & $6 \mathrm{~cm}$ & & $4 \mathrm{~cm}$ & $6 \mathrm{~cm}$ & \\
\hline \multirow{2}{*}{ BULLXcell } & 61.25 & 70.26 & $65.75^{A}$ & 70.14 & 71.24 & $70.69^{A}$ \\
\hline & \pm 2.34 & \pm 4.14 & \pm 2.54 & \pm 2.16 & $\pm \mathbf{3 . 1 1}$ & \pm 1.84 \\
\hline \multirow{2}{*}{$\mathbf{T C F}$} & 55.96 & 66.10 & $61.033^{A}$ & 61.61 & 66.44 & $64.03^{B}$ \\
\hline & \pm 2.41 & $\pm \mathbf{3 . 3 9}$ & \pm 2.33 & \pm 1.13 & $\pm \mathbf{0 . 9 8}$ & \pm 0.91 \\
\hline \multirow{2}{*}{ SMG } & 44.57 & 47.00 & $45.79^{B}$ & 28.33 & 45.85 & $37.09^{C}$ \\
\hline & \pm 2.74 & $\pm \mathbf{3 . 1 9}$ & \pm 2.06 & \pm 2.91 & $\pm \mathbf{3 . 8 8}$ & $\pm \mathbf{3 . 1 0}$ \\
\hline \multirow{2}{*}{ Overall means } & $53.933^{b}$ & $61.12^{a}$ & & $53.36^{b}$ & $61.17^{a}$ & \\
\hline & \pm 1.90 & \pm 2.75 & & \pm 3.56 & \pm 2.61 & \\
\hline
\end{tabular}

Means with different superscripts $\mathrm{A}, \mathrm{B}, \mathrm{C}$ between rows and $\mathrm{a}, \mathrm{b}$ between columns are significantly different at least $(\mathrm{P}<$ $0.01)$.

Table 6: Effect of different extenders and additives on sperm motility during various stages of freeze/ thaw processing of dog semen (Mean $\pm \mathrm{SE}$ ).

\begin{tabular}{|c|c|c|c|c|c|}
\hline Treatments & $\begin{array}{c}\text { After dil. } \\
\text { motility } \\
(\%)\end{array}$ & $\begin{array}{c}\text { Pre-freez } \\
\text { motility } \\
(\%)\end{array}$ & $\begin{array}{c}\text { Post- thaw } \\
\text { motility } \\
(\%)\end{array}$ & $\begin{array}{c}\text { Viability } \\
\text { index }\end{array}$ & $\begin{array}{l}\text { Post- thaw } \\
\text { recovery rate } \\
(\%)\end{array}$ \\
\hline BULLXcell & $\begin{array}{l}81.50^{\mathrm{A}} \\
\pm 1.29 \\
\end{array}$ & $\begin{array}{c}81.50^{\mathrm{A}} \\
\pm 1.29 \\
\end{array}$ & $\begin{array}{c}56.00^{\mathrm{AB}} \\
\pm 2.08 \\
\end{array}$ & $\begin{array}{c}134.00^{B} \\
\pm 6.68 \\
\end{array}$ & $\begin{array}{c}68.28^{B C} \\
\pm 2.32 \\
\end{array}$ \\
\hline TCF & $\begin{array}{c}82.00^{\mathrm{A}} \\
\pm 0.81 \\
\end{array}$ & $\begin{array}{c}82.00^{\mathrm{A}} \\
\pm 0.81 \\
\end{array}$ & $\begin{array}{c}51.50^{B} \\
\pm 1.49 \\
\end{array}$ & $\begin{array}{c}126.25^{\mathrm{B}} \\
\pm 5.38 \\
\end{array}$ & $\begin{array}{l}62.83^{C} \\
\pm 1.85 \\
\end{array}$ \\
\hline TCF- Eq & $\begin{array}{l}82.50^{\mathrm{A}} \\
\pm 0.83 \\
\end{array}$ & $\begin{array}{c}82.50^{\mathrm{A}} \\
\pm 0.83 \\
\end{array}$ & $\begin{array}{c}61.50^{\mathrm{A}} \\
\pm 1.29 \\
\end{array}$ & $\begin{array}{c}175.25^{\mathrm{A}} \\
\pm 8.31 \\
\end{array}$ & $\begin{array}{c}74.66^{\mathrm{AB}} \\
\pm 1.97 \\
\end{array}$ \\
\hline TCF- BHT & $\begin{array}{c}82.50^{\mathrm{A}} \\
\pm 0.83\end{array}$ & $\begin{array}{c}82.50^{A} \\
\pm 0.83\end{array}$ & $\begin{array}{c}59.00^{\mathrm{A}} \\
\pm 1.63\end{array}$ & $\begin{array}{r}158.00^{\mathrm{A}} \\
\pm 3.45\end{array}$ & $\begin{array}{c}71.47^{\mathrm{AB}} \\
\pm 1.66\end{array}$ \\
\hline TCF-Eq+ BHT & $\begin{array}{c}82.50^{\mathrm{A}} \\
\pm 0.83 \\
\end{array}$ & $\begin{array}{c}82.50^{\mathrm{A}} \\
\pm 0.83 \\
\end{array}$ & $\begin{array}{c}61.00^{A} \\
\pm 1.24 \\
\end{array}$ & $\begin{array}{c}175.00^{\mathrm{A}} \\
\pm 7.53 \\
\end{array}$ & $\begin{array}{c}77.82^{\mathrm{A}} \\
\pm 3.77 \\
\end{array}$ \\
\hline SMG & $\begin{array}{c}78.00^{\mathrm{AB}} \\
\pm 2.25 \\
\end{array}$ & $\begin{array}{c}78.00^{\mathrm{AB}} \\
\pm 2.25 \\
\end{array}$ & $\begin{array}{c}29.50^{\mathrm{DE}} \\
\pm 1.45 \\
\end{array}$ & $\begin{array}{c}43.75^{D} \\
\pm 7.07 \\
\end{array}$ & $\begin{array}{c}37.43^{\mathrm{EF}} \\
\pm 3.76\end{array}$ \\
\hline SMG-Eq & $\begin{array}{c}78.00^{\mathrm{AB}} \\
\pm 1.52 \\
\end{array}$ & $\begin{array}{c}78.00^{\mathrm{AB}} \\
\pm 1.52 \\
\end{array}$ & $\begin{array}{c}42.00^{C} \\
\pm 2.70 \\
\end{array}$ & $\begin{array}{c}84.00^{C} \\
\pm 9.78 \\
\end{array}$ & $\begin{array}{c}53.62^{\mathrm{D}} \\
\pm 2.93 \\
\end{array}$ \\
\hline SMG-BHT & $\begin{array}{l}76.00^{B} \\
\pm 1.94 \\
\end{array}$ & $\begin{array}{l}76.00^{B} \\
\pm 1.94 \\
\end{array}$ & $\begin{array}{c}25.00^{E} \\
\pm 3.33 \\
\end{array}$ & $\begin{array}{l}32.00^{D} \\
\pm 5.39 \\
\end{array}$ & $\begin{array}{l}32.42^{\mathrm{F}} \\
\pm 4.11 \\
\end{array}$ \\
\hline SMG- Eq+ BHT & $\begin{array}{l}75.50^{\mathrm{B}} \\
\pm 1.38 \\
\end{array}$ & $\begin{array}{c}75.50^{\mathrm{B}} \\
\pm 1.38 \\
\end{array}$ & $\begin{array}{c}33.50^{D} \\
\pm 2.11 \\
\end{array}$ & $\begin{array}{l}41.75^{D} \\
\pm 5.02 \\
\end{array}$ & $\begin{array}{c}44.40^{E} \\
\pm 2.63 \\
\end{array}$ \\
\hline Overall Means & $\begin{array}{r}79.83 \\
\pm 0.53\end{array}$ & $\begin{array}{r}79.83 \\
\pm 0.53\end{array}$ & $\begin{array}{l}46.55 \\
\pm 1.60\end{array}$ & $\begin{array}{r}107.77 \\
\pm 6.22\end{array}$ & $\begin{array}{l}58.10 \\
\pm 1.91\end{array}$ \\
\hline
\end{tabular}

Means with different superscripts $A, B, C, \ldots$ between rows are significantly different at least $(\mathrm{P}<0.01)$. 
Assiut Vet. Med. J. Vol. 61 No. 145 April 2015

Table 7: Effect of different extenders and additives on post-thaw percentage of unstained spermatozoa as well post-thaw acrosomal defects and acrosome reaction of cryopreserved of dog semen. (Mean \pm SE).

\begin{tabular}{|c|c|c|c|c|c|}
\hline \multirow[b]{2}{*}{$\begin{array}{l}\text { Semen } \\
\text { treatment }\end{array}$} & $\begin{array}{c}\text { Criteria of } \\
\text { PMI. }\end{array}$ & \multirow{2}{*}{$\begin{array}{c}\text { Unstained } \\
\text { spermatozoa } \\
(\%)\end{array}$} & \multirow{2}{*}{$\begin{array}{c}\text { Acrosomal } \\
\text { defects } \\
(\%)\end{array}$} & \multicolumn{2}{|c|}{$\begin{array}{l}\text { Acrosome } \\
\text { reaction }\end{array}$} \\
\hline & & & & $\begin{array}{c}\text { Complete } \\
(\%)\end{array}$ & $\begin{array}{c}\text { Incomplete } \\
(\%)\end{array}$ \\
\hline & BULLXcell & $\begin{array}{c}60.80^{\mathrm{B}} \\
+1.25\end{array}$ & $\begin{array}{c}36.80^{\mathrm{C}} \\
+1.87\end{array}$ & $\begin{array}{l}9.40^{C} \\
+0.30\end{array}$ & $\begin{array}{c}17.50^{\mathrm{CD}} \\
+0.16\end{array}$ \\
\hline & TCF & $\begin{array}{c}53.00^{\mathrm{DE}} \\
\pm 0.51\end{array}$ & $\begin{array}{c}42.00^{\mathrm{AB}} \\
\pm 0.51\end{array}$ & $\begin{array}{c} \pm 0.50 \\
14.70^{\mathrm{A}} \\
\pm 0.97\end{array}$ & $\begin{array}{c} \pm 0.10 \\
26.10^{A} \\
\pm 2.13\end{array}$ \\
\hline & TCF- Eq & $\begin{array}{c}67.90^{A} \\
\pm 0.68\end{array}$ & $\begin{array}{c}36.30^{C} \\
\pm 0.39\end{array}$ & $\begin{array}{l}8.80^{\mathrm{C}} \\
\pm 0.32 \\
\end{array}$ & $\begin{array}{l}11.80^{\mathrm{E}} \\
\pm 0.66\end{array}$ \\
\hline & TCF-BHT & $\begin{array}{c}54.80^{\mathrm{CD}} \\
\pm 1.62\end{array}$ & $\begin{array}{c}45.40^{A} \\
\pm 0.16\end{array}$ & $\begin{array}{c}11.70^{B} \\
\pm 0.90\end{array}$ & $\begin{array}{c}21.80^{B} \\
\pm 1.54\end{array}$ \\
\hline & TCF-Eq+ BHT & $\begin{array}{c}56.90^{\mathrm{C}} \\
\pm 1.53 \\
\end{array}$ & $\begin{array}{c}39.30^{B C} \\
\pm 1.71\end{array}$ & $\begin{array}{c}11.00^{\mathrm{BC}} \\
\pm 0.25\end{array}$ & $\begin{array}{c}17.50^{D} \\
\pm 0.16 \\
\end{array}$ \\
\hline & SMG & $\begin{array}{c}52.00^{\mathrm{DE}} \\
\pm 0.96\end{array}$ & $\begin{array}{c}42.60^{\mathrm{AB}} \\
\pm 0.74\end{array}$ & $\begin{array}{c}15.40^{\mathrm{A}} \\
\pm 1.10\end{array}$ & $\begin{array}{c}29.40^{\mathrm{A}} \\
\pm 1.10\end{array}$ \\
\hline & SMG-Eq & $\begin{array}{c}52.20^{\mathrm{DE}} \\
\pm 1.25\end{array}$ & $\begin{array}{c}45.60^{A} \\
\pm 0.16\end{array}$ & $\begin{array}{l}12.10^{B} \\
\pm 0.68\end{array}$ & $\begin{array}{c}18.30^{\mathrm{CD}} \\
\pm 1.67\end{array}$ \\
\hline & SMG-BHT & $\begin{array}{c}54.40^{\mathrm{CD}} \\
\pm 0.16\end{array}$ & $\begin{array}{c}44.00^{A} \\
\pm 0.77^{A}\end{array}$ & $\begin{array}{c}14.70^{A} \\
\pm 0.97\end{array}$ & $\begin{array}{l}22.50^{\mathrm{B}} \\
\pm 0.16\end{array}$ \\
\hline & SMG- Eq+ BHT & $\begin{array}{l}49.50^{\mathrm{E}} \\
\pm 1.38\end{array}$ & $\begin{array}{c}45.30^{A} \\
\pm 1.83\end{array}$ & $\begin{array}{c}16.80^{A} \\
\pm 0.83\end{array}$ & $\begin{array}{c}21.30^{\mathrm{BC}} \\
\pm 0.97\end{array}$ \\
\hline & Overall Means & $\begin{array}{l}55.72 \\
\pm 0.66\end{array}$ & $\begin{array}{l}41.92 \\
\pm 0.51\end{array}$ & $\begin{array}{l}12.73 \\
\pm 0.37\end{array}$ & $\begin{array}{l}20.68 \\
\pm 0.63\end{array}$ \\
\hline
\end{tabular}

Means with different superscripts $A, B, C, \ldots$ between rows are significantly different at least $(\mathrm{P}<0.01)$.

$\mathrm{PMI}=$ plasma membrane integrity

Table 8: Effect of different extenders and additives on rate of increase in post-thaw activity of extracellular AST, ALT and ALP Enzymes (Mean \pm SE).

\begin{tabular}{|c|c|c|c|}
\hline \multirow[b]{2}{*}{$\begin{array}{l}\text { Semen } \\
\text { Treatments }\end{array}$} & \multicolumn{3}{|c|}{$\begin{array}{l}\text { Rate of increase in post-thaw activity of extracellular } \\
(\%)\end{array}$} \\
\hline & AST & ALT & ALP \\
\hline BULLXcell & $\begin{array}{l}30.20^{\mathrm{E}} \\
\mathbf{+ 0 . 9 0}\end{array}$ & $\begin{array}{l}48.65^{\mathrm{Bc}} \\
\pm 2.17\end{array}$ & $\begin{array}{c}55.60^{\mathrm{B}} \\
\pm 1.80 \\
\end{array}$ \\
\hline TCF & $\begin{array}{l}49.95^{\mathrm{C}} \\
\pm 3.04 \\
\end{array}$ & $\begin{array}{l}50.80^{B} \\
\pm 2.47 \\
\end{array}$ & $\begin{array}{l}56.40^{B} \\
\pm 1.66 \\
\end{array}$ \\
\hline TCF- Eq & $\begin{array}{l}38.10^{D} \\
\pm 2.43 \\
\end{array}$ & $\begin{array}{c}41.70^{\mathrm{CD}} \\
\pm 1.07 \\
\end{array}$ & $\begin{array}{r}32.20^{D} \\
\pm 2.44 \\
\end{array}$ \\
\hline TCF- BHT & $\begin{array}{l}57.90^{B} \\
\pm 2.71 \\
\end{array}$ & $\begin{array}{l}45.60^{\mathrm{BCD}} \\
\pm 1.10\end{array}$ & $\begin{array}{l}46.20^{\mathrm{C}} \\
\pm 1.17 \\
\end{array}$ \\
\hline TCF-Eq+ BHT & $\begin{array}{c}29.60^{\mathrm{E}} \\
\pm 1.79 \\
\end{array}$ & $\begin{array}{c}38.50^{\mathrm{D}} \\
\pm 4.53 \\
\end{array}$ & $\begin{array}{l}23.00^{\mathrm{E}} \\
\pm 1.54 \\
\end{array}$ \\
\hline SMG & $\begin{array}{c}72.90^{\mathrm{A}} \\
\pm 1.23 \\
\end{array}$ & $\begin{array}{c}66.20^{A} \\
\pm 4.26 \\
\end{array}$ & $\begin{array}{c}79.50^{\mathrm{A}} \\
\pm \mathbf{1 . 3 8} \\
\end{array}$ \\
\hline SMG-Eq & $\begin{array}{c}42.30^{D} \\
\pm 2.58\end{array}$ & $\begin{array}{c}46.00^{\mathrm{BCD}} \\
\pm 1.63\end{array}$ & $\begin{array}{c}49.50^{\mathrm{C}} \\
\pm 1.38\end{array}$ \\
\hline SMG-BHT & $\begin{array}{c}62.00^{\mathrm{B}} \\
\pm 1.84\end{array}$ & $\begin{array}{l}59.00^{A} \\
\pm 2.08\end{array}$ & $\begin{array}{c}58.50^{\mathrm{B}} \\
\pm 1.38\end{array}$ \\
\hline SMG- Eq+ BHT & $\begin{array}{l}43.60^{D} \\
\pm 1.42\end{array}$ & $\begin{array}{c}49.60^{\mathrm{BC}} \\
\pm 3.03\end{array}$ & $\begin{array}{c}46.50^{\mathrm{C}} \\
\pm 1.38\end{array}$ \\
\hline Overall Means & $\begin{array}{l}47.39 \\
\pm 1.61\end{array}$ & $\begin{array}{l}49.56 \\
\pm 1.22\end{array}$ & $\begin{array}{r}49.71 \\
\pm 1.69\end{array}$ \\
\hline
\end{tabular}

Means with different superscripts $\mathrm{A}, \mathrm{B}, \mathrm{C}, \ldots$ between rows are significantly different at least $(\mathrm{P}<0.01)$. 
Assiut Vet. Med. J. Vol. 61 No. 145 April 2015

Table 9: Effect of different extenders and additives on DNA integrity using comet assay (Mean \pm SE).

\begin{tabular}{|c|c|c|c|}
\hline \multirow[b]{2}{*}{$\begin{array}{l}\text { Semen } \\
\text { Treatments }\end{array}$} & \multicolumn{3}{|c|}{ DNA integrity using comet assay } \\
\hline & $\begin{array}{c}\text { DNA Damage } \\
(\%)\end{array}$ & $\begin{array}{l}\text { Tail Length } \\
(\mu \mathrm{m})\end{array}$ & $\begin{array}{c}\text { Tail Moment } \\
\text { (Unit) }\end{array}$ \\
\hline BULLXcell & $17.45^{B} \pm 0.89$ & $9.20^{D} \pm 0.28$ & $2.05^{C} \pm 0.14$ \\
\hline TCF & $20.60^{B} \pm 0.46$ & $14.65^{\mathrm{BC}} \pm 0.20$ & $4.95^{B} \pm 0.14$ \\
\hline TCF- Eq & $17.35^{B} \pm 3.26$ & $12.60^{\mathrm{C}} \pm 2.13$ & $4.75^{B} \pm 0.02$ \\
\hline TCF- BHT & $20.50^{B} \pm 0.28$ & $12.65^{\mathrm{C}} \pm 0.31$ & $4.85^{B} \pm 0.14$ \\
\hline TCF-Eq+ BHT & $11.95^{\mathrm{C}} \pm 0.02$ & $8.10^{D} \pm 0.46$ & $1.60^{\mathrm{C}} \pm 0.23$ \\
\hline SMG & $29.00^{A} \pm 1.73$ & $18.35^{\mathrm{A}} \pm 0.25$ & $7.40^{\mathrm{A}} \pm 0.34$ \\
\hline SMG-Eq & $17.75^{B} \pm 3.03$ & $14.00^{\mathrm{C}} \pm 1.38$ & $4.90^{B} \pm 1.50$ \\
\hline SMG-BHT & $21.15^{B} \pm 0.54$ & $17.65^{\mathrm{A}} \pm 0.37$ & $7.25^{\mathrm{A}} \pm 0.14$ \\
\hline SMG- Eq+ BHT & $20.00^{B} \pm 0.57$ & $16.95^{\mathrm{AB}} \pm 0.60$ & $5.85^{\mathrm{AB}} \pm 1.24$ \\
\hline Overall Means & $19.52 \pm 0.95$ & $13.79 \pm 0.70$ & $4.84 \pm 0.41$ \\
\hline
\end{tabular}

Means with different superscripts $\mathrm{A}, \mathrm{B}, \mathrm{C}, \ldots$. between rows are significantly different at least $(\mathrm{P}<0.01)$.

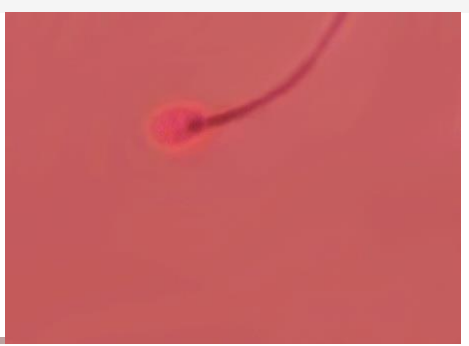

Dead spermatozoa

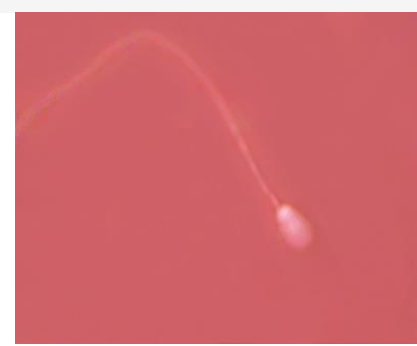

live spermatozoa

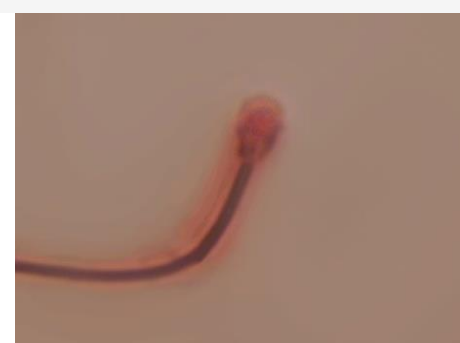

Acrosomal defect

Fig. 1: Posthaw live and dead spermatozoa as well as acrosomal defected obtained in German shepherd spermatozoa using Eosin stain exclusion assay

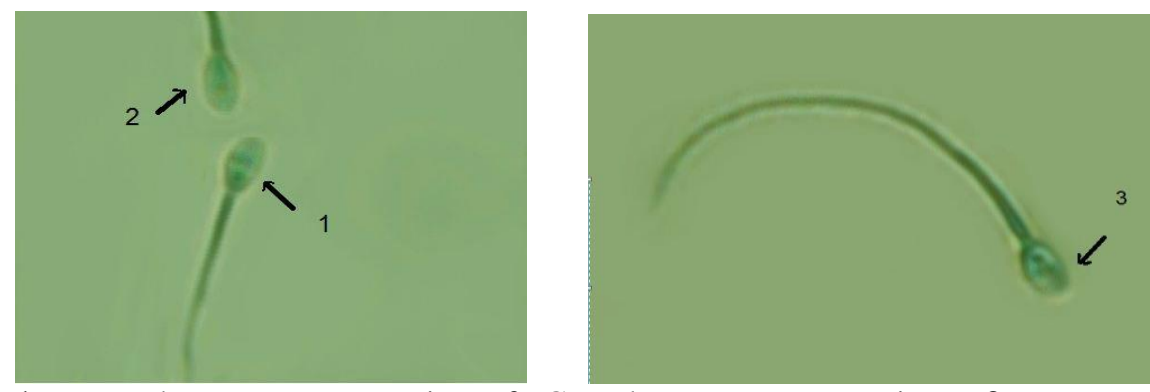

1: Incomplete acrosome reaction 2: Complete acrosome reaction $\quad 3$ : No acrosome reaction

Fig. 2: Postthaw acrosomal reaction obtained in German shepherd spermatozoa using silver nitrate stain

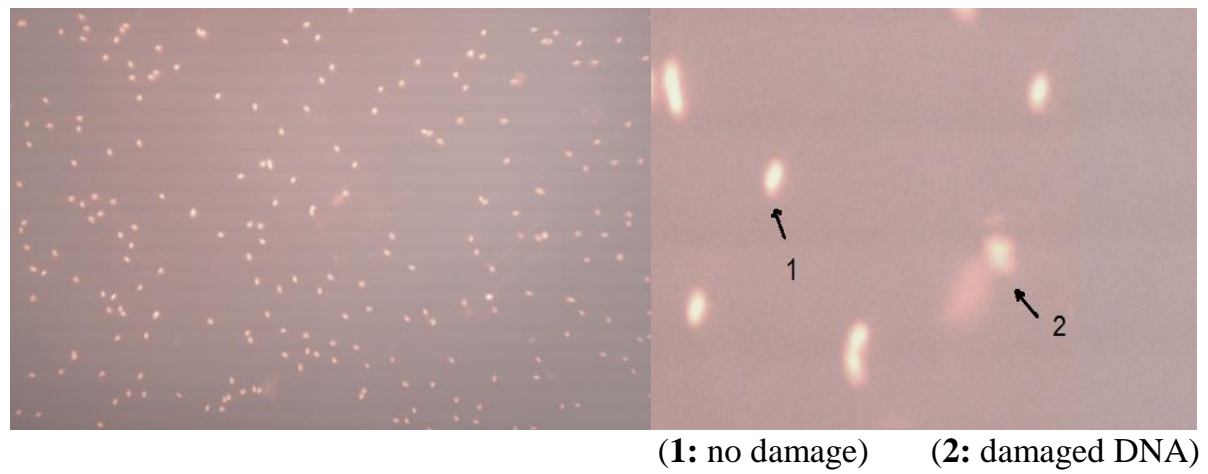

Fig. 3: Different Post thaw DNA damages (\%) obtained in German shepherd spermatozoa using comet assay 


\section{DISCUSSION}

The freezing-thawing process leads to an increase of the $\mathrm{O}_{2^{-}}$production. However, it remains to be documented if the increased concentrations of $\mathrm{O}_{2}$ - in canine semen are responsible for the alterations in the seminal parameters (Tselkas et al., 2000).

In the present study, post-thaw sperm motility, viability index and recovery rate were evidently improved significantly $(\mathrm{P}<0.01)$ after freezing of dog semen above liquid nitrogen surface by $6.00 \mathrm{~cm}$ and thawed at $55^{\circ} \mathrm{C}$ for $5 \mathrm{sec}(49.00 \pm 2.14 \%, 106.50 \pm$ 7.57 and $61.17 \pm 2.61 \%$, respectively) than those above liquid nitrogen surface by $4.00 \mathrm{~cm}(43.16 \pm$ $2.96 \%, 77.08 \pm 8.54$ and $53.36 \pm 3.56 \%$, respectively). While, results obtained with thawing rate $45^{\circ} \mathrm{C}$ for 30 $\sec (48.66 \pm 2.22 \%, 70.83 \pm 7.31$ and $61.12 \pm 2.75 \%$, respectively) for $6 \mathrm{~cm}$ than $4 \mathrm{~cm}$ height $(43.16 \pm 1.68$ $\%, 57.25 \pm 5.19$ and $53.93 \pm 1.90 \%$, respectively). In agreement with the obtained data, Baran et al. (2012) recorded that post thaw motility was $(48.54 \pm 8.27 \%$, $51.97 \pm 7.51 \%$, respectively) with freezing protocol 6 $\mathrm{cm}$ above liquid nitrogen and thawing regime $45^{\circ} \mathrm{C}$ for $60 \mathrm{sec}$ for canine spermatozoa extended in TCF and SMG extenders. Also, Hori et al. (2006) stated that horizontal placement of canine semen straws above $\mathrm{LN}_{2}$ to reduce the temperature at slow cooling rate of about $-10{ }^{\circ} \mathrm{C}$ followed by plunging into $\mathrm{LN}_{2}$ after sensitization 10-15 minutes provides good semen qualities after thawing.

Regarding to the thawing regimes, Ivanova-Kicheva et al. (1995) recorded that frozen canine semen samples in pellet and thawed at $55^{\circ} \mathrm{C}$ for $5 \mathrm{sec}$ vs. $37^{\circ} \mathrm{C}$ for $8 \mathrm{sec}$, showed significantly post thaw motility, survival time and lower acrosomal damage although the percentage of abnormalities not found to be different between two thawing regimens. In another studies, Seager and Platz (1977); Battista et al. (1989), Olar et al. (1989); Rota et al. (1998) and Nöthling and Shuttleworth, 2005) attributed the greater post-thaw motility after freezing in straws resulted from rapid thaw rates of $70^{\circ} \mathrm{C}$ for $6 \mathrm{sec}$ or 45 ${ }^{\circ} \mathrm{C}$ for $30 \mathrm{sec}$ compared to slow rates of $37^{\circ} \mathrm{C}$ for $50 \mathrm{sec}$ or $23^{\circ} \mathrm{C}$ for several minutes. On the contrary, both Smith and Graham (1984) and Yubi et al. (1987) found, after freezing semen in straws, slower thaw rates $\left(37^{\circ} \mathrm{C}\right.$ for $\left.2 \mathrm{~min}\right)$ resulted in the best post-thaw semen quality, with a decline in motility and percent live sperm after rapid thawing $\left(75^{\circ} \mathrm{C}\right.$ for $\left.6 \mathrm{sec}\right)$.

Nearly similar results in goat was detected by Bezerra et al. (2012) who recorded optimal results for progressive motility were achieved when goat semen was frozen in $0.5 \mathrm{~mL}$ straws and thawed in water at $55^{\circ} \mathrm{C}$ for $7 \mathrm{sec}$. On the other hand, studies have demonstrated that a fast thawing rate results in better post-thaw quality when compared with slower thawing for stallions, rams (Watson, 1990), bulls
(Pace et al., 1981), boars (Eriksson and RodriguezMartinez, 2000).

Concerning the possible explanation for the favourable effect of thawing at high temperature Lahnsteiner (2000) recommended performing the thawing at high temperatures in order to avoid recrystallization, because the warming damage occurs when the spermatozoa pass through the critical zone of -50 through $-15{ }^{\circ} \mathrm{C}$ or $-5{ }^{\circ} \mathrm{C}$. Similarly, spermatozoa suffer osmotic stress, when the duration of thawing is insufficient for the outflow of excess cryoprotectants from the cell and the spermatozoa swells and lyses as the medium dilutes abruptly on account of melting of the extracellular ice (Andrabi, 2007).

Under the present experimental condition, inclusion of $0.5 \%$ Equex STM paste and /or 1mM BHT in Tris based extenders resulted in pronounced improvement in the post-thaw sperm motility, viability index, recovery rate and plasma membrane integrity in terms of post-thaw live spermatozoa, acrosomal defects, acrosome reaction and rate of increase in post-thaw activity of extracellular (AST, ALT and ALP) enzymes as well as assessment of sperm DNA integrity.

Regarding to the impact of Equex STM paste on the spermatozoa function, the present study pointed out the supplementation of dog semen extender with $0.5 \%$ of Equex STM paste in Tris and milk based extenders resulted in a pronounced improvement in the motility, viability, acrosomal membrane stability, plasma membrane as well sperm DNA integrity. In agreement with our data Scha"fer-Somi et al. (2006) concluded that best post-thaw motility and longevity were achieved with a two-step dilution, the second phase extender containing Equex STM paste, deep freezing in the box and thawing at $70{ }^{\circ} \mathrm{C}$ for $8 \mathrm{sec}$. Also, Spermatozoa membrane damages was less pronounced when the extender contained Equex STM paste. The membrane stabilizing effect of this detergent containing paste, increasing post- thaw longevity, has been shown in many studies Thomas et al. (1992); Rota et al. (1997); Pena and LindeForsberg (2000b); Pena et al. (2003) and Rodenas et al. (2014). Moreover, the present investigation clarified that Equex STM paste supplementation induced a significant improvement in post-thaw acrosomal membrane stability. Nearly similar effect was detected by Petrunkina et al. (2005); Ponglowhapan and Chatdarong (2008) and Farstad (2012).

As regards the possible explanation for the beneficial influence of Equex STM paste on the functional competence of frozen- thawed dog spermatozoa, a variety of postulates in the published literature were focused on the ability of Equex STM paste to minimize the undesirable destabilization of sperm 
membranes by the active compound of Equex STM paste, sodium dodecyl sulfate (SDS), was thought to act through alteration of the egg yolk by solubilization of protecting egg yolk-lipids increasing the egg yolk's protection potential against cold shock and freezing injury (Pursel et al., 1978, Arriola and Foote 1987; Penfold and Moore 1993). Also, SDS seems to modify membrane fluidity and thus minimizes the occurrence of capacitation-like changes initiated during cryopreservation in ram semen (Watson 1995) and protect against redistribution of proteins at other locations and aggregation in fewer sites which induced gaps in membrane integrity and resultant leaks as well as hampered function of enzymes or receptors (Amann, 1999). The protecting effect against lipid phase transitions was confirmed for dog spermatozoa (Peña and Linde-Forsberg 2000b) including partial protection of functionality of the plasma membrane Ca2+-pumps. The pumps are damaged but still can hold the $\mathrm{Ca} 2+-$ concentration under a certain threshold, which usually triggers the false acrosome reaction and cell death. So, the protective effect of Equex STM paste on dog spermatozoa was found to be more pronounced if it was added only immediately before freezing, suggesting that prolonged exposure to SDS may exert a direct negative effect on sperm membranes and that the beneficial effect of SDS may depend on concentration and time of exposure (Peña and Linde-Forsberg, 2000b). On the contrary, Bateman (2001) found neither an obvious benefit immediately post-thaw nor any detrimental effects on sperm motility, morphology, acrosomal status with supplementation of $1 \%$ Equex STM paste in canine semen frozen in pellets. In the same line, the addition of different amounts of Equex STM paste for the freezing of semen has been found to be beneficial in the boar (Pursel et al., 1978), stallion (Martin et al., 1979) and mouse (Penfold and Moore1993).

Concerning the influence of BHT on the functional competence of frozen- thawed dog spermatozoa, the current study indicated that inclusion of $1 \mathrm{mM}$ BHT in Tris based extenders caused a significant improvement in motility, viability, acrosomal integrity, plasma membrane integrity as well as sperm DNA integrity. These results are in agreement with a previous study of for Neagu et al. (2010); Sahashi et al. (2011) and Ziaullah et al. (2012) who reported that BHT was found to improve significantly all postthawed canine semen quality parameters in terms of sperm motility, acrosomal integrity, hypo-osmotic swelling response, membrane integrity and viability at an inclusion level of $1 \mathrm{mM}$ in the extended semen. BHT has been used successfully for preservation of liquid semen in turkey tom (Donoghue and Donoghue, 1997) and to minimize cryoinjury in ram (Watson and Anderson, 1983), boar (Roca et al., 2004), cattle bull (Shoae and Zamiri, 2008), goat spermatozoa (Khalifa et al., 2008 and Naijian et al., 2013) and human spermatozoa (Merino et al., 2015).
The optimal inclusion level for BHT in results seems to be species specific characteristics may be due to variability in concentration of spermatozoa used.

Since sperm membrane is rich in polyunsaturated fatty acids, it can easily undergo lipid peroxidation during sperm processing and storage in the presence of reactive oxygen species (ROS). Peroxidation of sperm membrane phospholipids leading to change in membrane fluidity then results in loss of motility. Finally, the peroxidized sperm cells lack the membrane dynamics of fusogenicity and are deficient in fertilization of oocytes (Alvarez and Storey, 1992). Concerning the possible explanation for the protective effect of antioxidant (BHT) is attributed to two mechanisms: firstly, the incorporation of the compound in the sperm membranes, hence, making them more fluidic and preventing them from the damage (Ijaz et al., 2009) and, secondly, declining the damaging potential of lipid peroxyl radicals by conversion into hydroperoxides (Thomassen et al., 2006).

Under the present experimental condition, the best post -thaw semen parameters was obtained with occlusion of Equex STM paste and or BHT in Tris based extender than skim milk based extender. Nearly similar results was reported by Neagu et al. (2010) who recorded Uppsala extender (tris based extender containing Equex STM paste) was superior to INRA plus glycerol (milk-based, ready-to-use conservation extender for stallion semen) in post-thaw membrane integrity. Also, Graham and Hammerstedt (1992) who reported that BHT analogues protected bull spermatozoa from cold shock in egg yolk-based extenders but not in skim milk-based extenders. However, significantly better results were observed in controls using Uppsala extender. The possible explanation may be due to the interactions between the components of the extender and the antioxidant, however, must be kept into account. As BHT is lipidsoluble, it may remain associated with egg yolk lipids hence scanty free BHT may be available for permeating the sperm plasma membrane (Killian et al., 1989). Also, Hermansson and Linde-Forsberg (2006) and Santana et al. (2013) observed that postthaw motility was slightly lower in milk based extender than that described for TEY diluent after 24 $\mathrm{h}$ cooled storage while, membrane and acrosome integrity were within the range.

Concerning the effect of Equex STM paste and or BHT supplementation on the sperm DNA integrity, the current study revealed these two additives could reduce the effect of sperm DNA damage which promoted by cryopreservation in dog. In agreement with our data (Kim et al., 2010) cryopreserved dog semen in Tris based extender containing 0.5\% Equex STM paste and frozen at $4 \mathrm{~cm}$ above the level of $\mathrm{LN}_{2}$. He recorded that cryopreservation caused sperm PS 
translocation, intracellular $\mathrm{H}_{2} \mathrm{O}_{2}$ production of viable sperm, and DNA fragmentation due to ROS resulting from membrane lipid peroxidation which directly damage sperm DNA assay (Baumber et al., 2003). This may be one of the major factors to explain reduction of fertility in frozen-thawed canine semen The DNA damage of frozen-thawed canine semen in the present study is in the normal range $(<30 \%)$ as recorded by Kim et al. (2010). The latter authors suggested that the DNA assessment should be an additional and complementary parameter to better assess sperm quality after freezing thawing in canine.

With respect to the effect of Equex STM paste plus BHT extenders, the current investigation indicated that supplementation of Tris based extenders with 0.5 $\%$ Equex STM paste plus $1 \mathrm{mM}$ BHT resulted significantly provoked an improvement in postthaw motility, viability, plasma membrane integrity, acrosomal membrane stability and sperm DNA integrity. Similar effects were achieved for by Neagu et al. (2010). The possible explanation for that augmented improvement may be due to the synergistic action of both additives on canine spermatozoa.

With respect to commercial bovine tris based semen extender (BULLXcellTM, IMV technologies, Franc). The BULLXcell extender is useful for cryopreservation of dog semen. Regarding to its effect on the spermatozoa function, the present study resulted in a pronounced improvement in the motility, viability, acrosomal membrane stability, plasma membrane as well sperm DNA. In the parallel line, Szász et al. (2000) and Martins et al. (2012) found that commercial bovine semen extender TRILADYL and Bovimix(®) respectively were useful for cryopreservation of dog semen.

It is concluded that fortification of $0.5 \%$ Equex STM paste and / or $1 \mathrm{mM}$ BHT in Tris based extenders freezing above $6 \mathrm{~cm}$ highest above $\mathrm{LN}_{2}$ and thawing at $55{ }^{\circ} \mathrm{C}$ for $5 \mathrm{sec}$. can improve the post-thaw dog semen motility, viability and plasma membrane integrity. Also, the commercial bovine extender BULLXcell ${ }^{\mathrm{TM}}$ extender is useful for cryopreservation of dog semen.

\section{REFERENCES}

Alamo, D.; Batista, M.; Gonzalez, F.; Rodriguez, N.; Cruz, G.; Cabrera, F. and Gracia, A. (2005): Cryopreservation of semen in the dog: use of ultra-freezers of $-152^{\circ} \mathrm{C}$ as available alternative to liquid nitrogen. Theriogenology, 63: 72-82.

Alvarez, J.G. and Storey, B.E. (1992): Evidence for increased lipid peroxidative damage and loss of superoxide dismutase activity as a mode of sublethal cryodamage to human sperm during cryopreservation. J. Androl., 13: 232-241.
Amann, R.P. (1999): Cryopreservation of sperm. In Encyclopedia of Reproduction: E. KNOBIL and J.D. NEILL (eds.): New York, Academia Press $1,773-783$

Andrabi, S.M.H. (2007): Fundamental principles of cryopreservation of Bos taurus and Bos indicus bull spermatozoa. Int. J. Agri. Biol., 9(2): 367-369.

Arriola, J. and Foote, R.H. (1987): Glycerolisation and thawing effects on bull spermatozoa frozen in detergent treated egg yolk and whole egg extenders. J. Dairy Sci., 70: 1664-1670.

Baran, A.; Ak, K. and Ileri, I.K. (2000): Freezing of dog semen in pellets and straws with tris and milk extenders. Vet. Fakultesi Dergisi Istanbul, 26: 251-263.

Baran, A.; Qzdas, O.B.; Sandal, A.I. and Kemal, A.K. (2012): Effect of skim milk and tris extenders on frozen thawed canine sperm morphology. J. Vet. Anim. Adv., 11 (17): 3242- 3246.

Bateman, H.L. (2001): Effects of semen extender composition and cooling methods on canine sperm function and cryosurvival. Univ. of Guelph, M. Sc. Ref. in: Diss. Abstr. Int., 4142, 57-76

Battista, M.; Parks, J. and Concannon, P. (1989): Canine sperm post-thaw survival following freezing in straws or pellets using PIPES. lactose, Tris or Test extenders. Anim. Reprod. Sci., 3: 229-231.

Baumber, J.; Ball, B.A.; Linfor, J.J. and Meyers, S.A. (2003): Reactive oxygen species and cryopreservation promote DNA fragmentation in equine spermatozoa. J. Andro., 1 (24): 621-628.

Belfield, A. and Goldberg, D.M. (1971): Hydrolysis of adenosine monophosphates by acid phosphatases as measured by a continuous spectrophotometric assay. Biochem. Med. Sep., 4 (2): 135-148.

Bezerra, F.S.B.; Castelo, T.D.S.; Santos, E.A.A.; Dantas, T.D.C.; Simão, B.R. and Silva, A.R. (2012): Assessment of the interaction between straw size and thawing rate and its impact on in vitro quality of post-thaw goat semen. R. Bras. Zootec., 41 (3): Viçosa Mar. 2012. Online version ISSN 1806-9290.

Collins, A.R.; Ai-guo, M. and Dothie, S.J. (1995): The kinetics of repair of oxidative DNA damage (strand breaks and oxidized pyrimidines) in human cells. Mut. Res., 336: 69-77.

Costat Computer Program Copyright (1986): Version 3:03 copyright softwar.

Donoghue, A.N. and Donoghue, D.J. (1997): Effects of water and lipid-soluble antioxidants on turkey sperm viability, membrane integrity, and motility during liquid storage. Poultry Science, (76): 1440-1445. 
El-Amrawi, G.A. and Nemetallah, B.A. (1991): Study on capacitation of buffalo spermatozoa. Egypt. Soc. Anim. Reprod. Fertil., 12-14: 214.

Eriksson, B.M. and Rodriguez-Martinez, H. (2000): Effect of freezing and thawing rates on the post-thaw viability of boar spermatozoa frozen in Flat Packs and Maxi-straws. Anim. Reprod. Sci., 63(3-4): 205-220.

Farstad, W. (2012): Customizing semen preservation protocols for individual dogs and individual species: sperm preservation beyond the state of the art. Reprod. Domest. Anim., 47 Suppl 6:269-273.

Graham, J.K. and Hammerstedt, R.H. (1992): Differential effects of butylated hydroxytoluene analogs on bull sperm subjected to cold-induced membrane stress. Cryo., 29 (1): 106-117.

Hermansson, U. and Linde Forsberg, C. (2006): Freezing of stored, chilled dog spermatozoa. Theriogenology, 65 (3): 584-593.

Hori, T.; Odaka, S.; Oba, H.; Mizutani, T.; Kawakami, E. and Tsutsui, T. (2006): Effects of liquid nitrogen vapor sensitization conditions on the quality of frozen-thawed dog spermatozoa. J. Vet. Med. Sci., 68 (10): 1055-1061.

Hughes, C.M.; Lewis, S.E.; McKelvey-Martin, V.J. and Thompson, W. (1997): Reproducibility of human sperm DNA measurements using the alkaline single cell gel electrophoresis assay. Mut. Res., 374: 261-268.

Ijaz, A.; Hussain, A.; Aleem, M.; Yousaf, M.S. and Rehman, M. (2009): Butylated hydroxytoluene inclusion in semen extender improves the postthawed semen quality of Nili-Ravi buffalo (Bubalus bubalis). Theriogenology, 71: 1326-1329.

Ivanova-Kicheva, M.G.; Subev, M.S.; Bobadov, N.D.; Dacheva, D.P. and Rouseva, I.A. (1995): Effect of thawing regimens on the morphofunctional state of canine spermatozoa Theriogenology, 44, 4: 563-569.

Khalifa, T.A.A.; Lymberopoulos, A.G. and El-Saidy, B.E. (2008): Testing usability of butylated hydroxytoluene in conservation of goat semen. Reprod. Dom. Anim., (43): 525-530.

Killian, G.; Honadel, T.; McNutt, T.; Henault, M.; Wegner, C. and Dunlap, D. (1989): Evaluation of butylated hydroxytoluene as a cryopreservative added to whole or skim milk diluent for bull semen. J. Dairy Sci., 72: 1291-1295.

Kim, S.H.; Yu, D.H. and Kim, Y.J. (2010): Effects of cryopreservation on phosphatidylserine translocation, intracellular hydrogen peroxide, and DNA integrity in canine sperm. Theriogenology,73(3): 282-292.

Kutzler, M.A. (2005): Semen collection in the dog. Theriogenology, 64: 747-754.
Lahnsteiner, F. (2000): Semen cryopreservation in the salmonidae and in the northern pike. Aquaculture Research, 31(3): 245-258.

Linde Forsberg, C. (2005): Artificial Insemination. In ESAVS-EVSSAR Course Reproduction in companion, exotic and laboratory animal.

Linde-Forsberg, C.; Ström Holst, B. and Govette, G. (1999): Comparison of fertility data from vaginal vs intrauterine insemination of frozenthawed dog semen: A retrospective study Original Research Article Theriogenology, 52(1): 11-23.

Martin, J.C.; Klug, E. and Gunzel, A.R. (1979): Centrifugation of stallion semen and its storage in large volume straws. J. Reprod. Fertil., (Suppl) 27: 47-51.

Martins, M.; Justino, R.C.; Sant'anna, M.C.; Trautwein, L.G. and Souza, F.F. (2012): Comparison of two different extenders for cryopreservation of epididymal dog sperm. Reprod. Domest. Anim., 47 Suppl 6: 293-294.

Merino, Q.; Aguaguina, W.E.: Esponda, P.; Risopatron, J.; Lsachenko, E. and Lsachenko, $R$. and Sanchez, V. (2015): protective effect ob butylated hydroxytoluene on sperm function in human cryopreserved by vitrification technique. Andrologia, 47 (2): 186-193.

Michael, A.; Alexopoulos, C.; Pontiki, E.; Hadjipavlou-Litina, D.; Saratsis, P. and Boscos, C. (2007): Effect of antioxidant supplementation on semen quality and reactive oxygen species of frozen-thawed canine spermatozoa. Theriogenology, 68(2): 204-212.

Milovanov, V.K. (1962): Biology of reproduction and artificial insemination of farm animals. Monograph. Selkhoz. Lit. J. and Plakatov, Moscow.

Naijian, H.R.; Kohram, H.; Shahneh, A.Z.; Sharafi, M. and Bucak, M.N. (2013): Effects of different concentrations of BHT on microscopic and oxidative parameters of Mahabadi goat semen following the freezethaw process. Original Research Article Cryobiology, 66(2): 151-155.

Neagu, V.R.; García, B.M.; Sandoval, C.S.; Rodríguez, A.M.; Ferrusola, C.O.; Fernández, L.G.; Tapia, J.A. and Peña, F.J. (2010): Freezing dog semen in presence of the antioxidant butylated hydroxytoluene improves post-thaw sperm membrane integrity. Theriogenology, 73(5): 645-650.

Nöthling, J.O. and Shuttleworth, R. (2005): The effect of straw size, freezing rate and thawing rate upon post-thaw quality of dog semen. Theriogenology, 63(5): 1469-1480.

Okano, T.; Murase, T.; Asano, M. and Tsubota, T. (2004): Effects of final dilution rate, sperm concentration and times for cooling and glycerol equilibration on post-thaw 
characteristics of canine spermatozoa. J. Vet. Med. Sci., 66(11): 1359-1364.

Olar, R.; Bowen, A. and Pickett, B.W. (1989): Influence of extender, cryoperservative and seminal processing procedures on post-thaw motility of canine spermatozoa frozen in straws. Theriogenology, 31(2): 451-461.

Pace, M.M.; Sullivan, J.J. and Elliott, F.I. (1981): Effects of thawing temperature, number of spermatozoa and spermatozoa quality on fertility of bovine spermatozoa packaged in $0.5 \mathrm{~mL}$ French straws. J. Anim. Sci., 53: 693-701.

Pan, S.W.; Liu, H. and Ma, D.J. (1986): Semen freezing technique of dog Anim. Husbandry and Vet. Me., 2 (3): 105-106.

Peña, A. and Linde-Forsberg, C. (2000a): Effects of spermatozoal concentration and post-thaw dilution rate on survival after thawing of dog spermatozoa. Theriogenology, 54(5): 703-718.

Peña, A. and Linde-Forsberg, C. (2000b): Effects of Equex, one- or two-step dilution, and two freezing and thawing rates on post-thaw survival of dog spermatozoa. Theriogenology, 54(6): 859-875.

Peña, A.I.; López-Lugilde, L.; Barrio, M.; Becerra, J.J.; Quintela, L.A. and Herradón, P.G. (2003): Studies on the intracellular $\mathrm{Ca} 2+$ concentration of thawed dog spermatozoa: influence of Equex from different sources, two thawing diluents and post-thaw incubation in capacitating conditions. Reprod. Domest. Anim., 38(1): 27-35.

Penfold, L.M. and Moore, H.D.M. (1993): A new method for cryopreservation of mouse spermatozoa. J. Reprod. Fertil., 99, 131-134

Petrunkina, A.M.; Gröpper, B.; Töpfer-Petersen, E. and Günzel-Apel, A.R. (2005): Volume regulatory function and sperm membrane dynamics as parameters for evaluating cryoprotective efficiency of a freezing extender. Theriogenology, 63(5): 1390-1406.

Ponglowhapan, S. and Chatdarong, K. (2008): Effects of Equex STM Paste on the quality of frozen-thawed epididymal dog spermatozoa Theriogenology, 69(6): 666-672.

Pursel, V.G.; Schulman, L.L. and Johnson, L.A. (1978): Effect of Orvus ES Paste on acrosome morphology, motility and fertilizing capacity of frozen-thawed boar sperm. J. Anim. Sci., 47: 198-202.

Reitman, S. and Frankel, S.A. (1957): Colorimetric method for the determination of serum glutamic oxalacetic and glutamic pyruvic transaminases. Amer. J. Clin. Pathol., 28: 56-63.

Roca, J.; Gil, M.A.; Hernandez, M.; Parrilla, I.; Vazquez, J.M. and Martinez, E.A. (2004): Survival and fertility of boar spermatozoa after freeze thawing in extender supplemented with butylated hydroxytoluene. J. And., (25): 397-405.

Rodeanas, C.; Parrilla, I.; Roca, J.; Martinez, E.A. and Lucas, X. (2014): Effect of rapid cooling prior to freezing on the quality of canine cryopreserved spermatozoa. J. Reprod. Dev., 60 (5): 355-361.

Rota, A.; Ström, B.; Linde-Forsberg, C. and Rodriguez-Martinez, H. (1997): Effects of equex STM paste on viability of frozen-thawed dog spermatozoa during in vitro incubation at $38{ }^{\circ} \mathrm{C}$ Theriogenology, 47(5): 1093-1101.

Rota, A.; Linde-Forsberg, C.; Vannozzi, J.; Romagnoli, S. and Rodriguez-Martinez, $H$. (1998): Cryosurvival of dog spermatozoa at different glycerol concentrations and freezing/thawing rates. Reprod. Dom. Anim., 33: 355-361.

Rowson, L.E.A. (1954): Infertility in cow, sow and bitch. Irish Veterinary Journal, 8 (10): 216-227.

Sahashi, Y.; Otsuki, T.; Higaki, S.; Nagano, M.; Yamashita, Y. and Hishinuma, M. (2011): Effect of butylated hydroxy-toluene on dog sperm longevity in chilling storage and cryopreservation. J. Vet. Med. Sci., (73): 895-899.

Santana, M.; Batista, M.; Alamo, D.; Gonzalez, F.; Niño, T.; Cabrera, F. and Gracia, A. (2013): Influence of cool storage before freezing on the quality of frozen-thawed semen samples in dogs. Reprod. Domest. Anim., 48: 165-170.

Schäfer-Somi, S.; Kluger, S.; Knapp, E.; Klein, E. and Aurich, C. (2006): Effects of semen extender and semen processing on motility and viability of frozen-thawed dog spermatozoa. Theriogenology, 66(2): 173-182.

Seager, S.W.J. and Platz, C.C. (1977): Collection and evaluation of canine semen. Symposium of Reproductive Problems, Veterinary Clinics of North America, 7, 765-773

Shoae, A. and Zamiri, M.J. (2008): Effect of butylated hydroxytoluene on bull spermatozoa frozen in egg yolk-citrate extender. Anim. Reprod. Sci., (104): 414-418.

Singh, N.P.; McCoy, M.T.; Tice, R.R. and Schneider, E.L. (1988): A simple technique for quantitation of low levels of DNA damage in individual cells. Exp. Cell Res. 175:184-191.

Smith, F.O. and Graham, E.F. (1984): Cryopreservation of canine semen: Technique and performance. $10^{\text {th }}$ Int. Congr. Anim. Reprod. A.I., 2: 216.

Szász, F.; Gábor, G. and Solti, L. (2000): Comparative study of different methods for dog semen cryopreservation and testing under clinical conditions. Acta Vet. Hung., 48 (3): 325-333.

Thomas, P.G.A.; Surman, V.; Myers-Wallen, V.N.; Concannon, P.W. and Ball, B.A. (1992): 
Addition of sodium dodecyl sulphate to the TRIS citrate extender improves motility and longevity of frozen thawed canine spermatozoa. Proc $12^{\text {th }}$ Int. Congr. Anim. Reprod. (ICAR) 1992; 4: 1823-1825

Thomassen, G.; Sanson, A.; Krogenas, J.A.; Fougner, K.; Andersen, B. and FarstadIn, W. (2006): Artificial insemination with frozen semen in dogs: A retrospective study of 10 years using a non-surgical approach. Theriogenology, 66 (6-7):, 1645-1650R.

Tselkas, K.; Saratsis, P.; Karagianidis, A. and Samouilidis, S. (2000): Extracellular presence of reactive oxygen species (ROS) in fresh and frozen-thawed canine semen and their effects on some semen parameters. Dtsch. Tierarztl. Wochenschr., 107(2): 69-72.

Vazquez, J.M.; Martinez, E.A.; Martinez, P.; GarciaArtiga, C. and Roca, J. (1997): Hypoosmotic swelling of boar spermatozoa compared to other methods for analysing the sperm membrane. Theriogenology, 47: 913.

Wang, L.B.; Yang, D.J. and Zhou, X.Y. (1991): Study on semen freezing of $\operatorname{dog}$ and artificial insemination. Heilongjiang Anim. Sci. And Vet. Med., 5: 35-36.

Wang, Y.; Sharma, R.K. and Agarwal, A. (1997): Effect of cryopreservation and sperm concentration on lipid peroxidation in human semen. Urology 50: 409-413.
Wang, X.L.; Liu, Y. and Zeng, S.M. (2008): Advance in international development of canine semen cryopreservation. Chinese J. Anim. Sci, 2(15): 12-16.

Watson, P. and Anderson, W. (1983): Influence of butylated hydroxytoluene (BHT) on the viability of ram spermatozoa undergoing cold shock. J. Reprod. and Fert., 69, 229-235.

Watson, P.F. (1990): Artificial insemination and the preservation of semen. in: Lamming GE (ed.) MarshaIl's physiology of reproduction, $4^{\text {th }} \mathrm{cd}$. vol 2. Edinburgh: Churchill Livingston,: 747-869.

Watson, P.F. (1995): Recent developments and concepts in the cryopreservation of spermatozoa and the assessment of their postthawing function. Reprod. Fertil. Dev., 7, 871-891.

Young, D.S. (1990): Effects of drugs on clinical laboratory tests. Third ed., 3: 6-12.

Yubi, A.C.; Ferguson, J.J.; Renton, M.P.; Harker, S.; Harvey, M.J.A.; Bagyenji, B. and Douglas, T.A. (1987): Some observations on the dilution, cooling and freezing of canine semen. J. Small Anim. Pract. 28, 753-761.

Ziaullah, M.; Ijaz, A.; Aleem, M.; Mahmood, A.K.; Rehman, H.; Bhattil, S.; Farooq, U. and Sohail, M.U. (2012): Optimal inclusion level of butylated hydroxytoluene in semen extender improves the quality of post-thawed canine sperm. Czech J. Anim. Sci., 57, (8): 377-381.

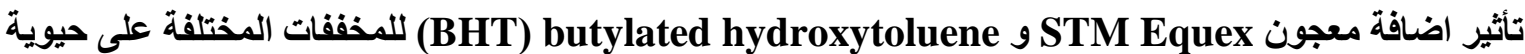 وسلامة أغثية البلازما والحمض النووى لحيامن كلاب الجيرمان شبرد أثناء التجميد ومعدلات الاسالة فى مصر على على
}

\author{
مارى جاد عبل الملاك ، احد سعبل محمود ، فكرى محمد حسبن ، كمال كمال متولح \\ Email: dr.marygad@hotmail.com Assiut University web-site: www.aun.edu.eg
}

هناك العديد من العوامل التي تؤثر على جودة السائل المنوي من خلال خطوات التجميد مثل تكوين المخففات المستخدمة، معدل التبريد وكذلك تقنية الاسالة. وقد أجريت هذه الدر اسة لتقييم تأثير اضافة معجون STM Equex و (BHT) butylated hydroxytoluene

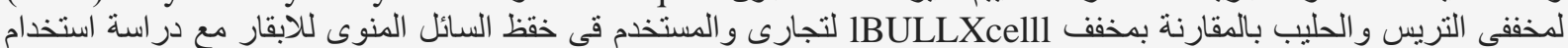

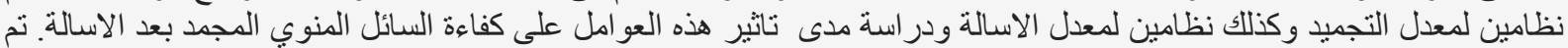

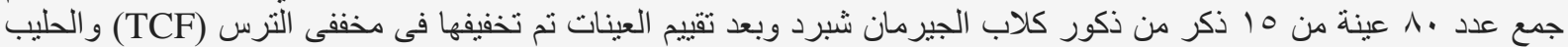

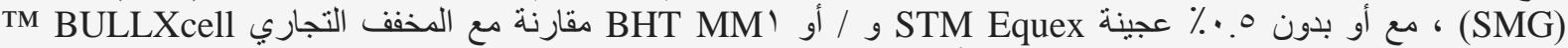

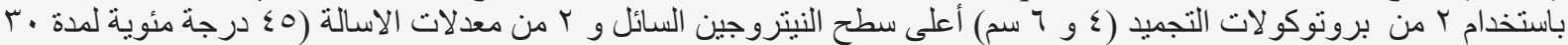

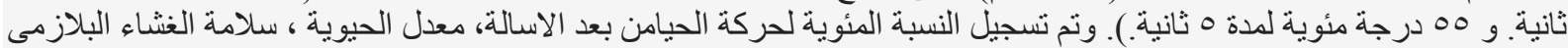

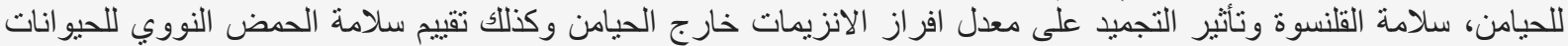

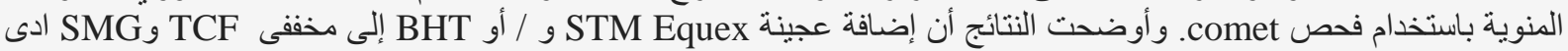

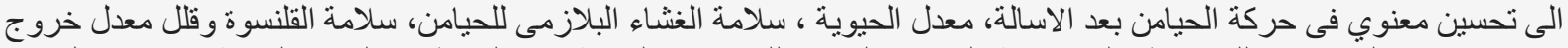

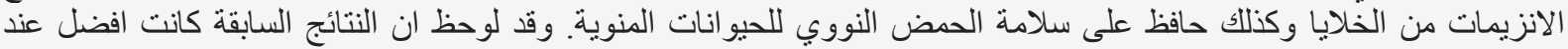

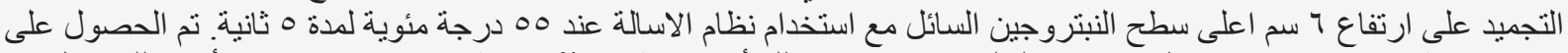

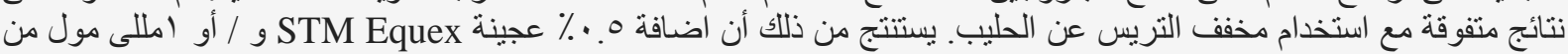

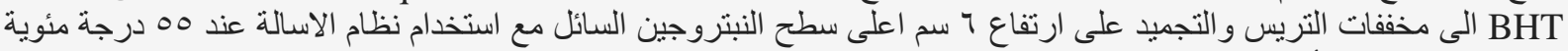

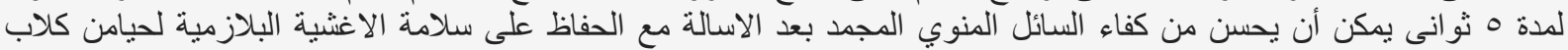

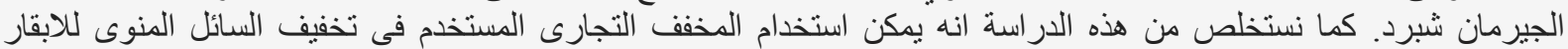
Tبامان في حفظ السائل المنوي للكلاب. 